\title{
Selected papers about chemiluminescence of flames
}

\author{
A. Brockhinke - C. Schulz
}

Published online: 30 May 2012

(C) Springer-Verlag 2012

Sensors that allow cheap, reliable and non-intrusive monitoring and subsequent active control of combustion processes are necessary for the development of combustors operating under potentially unstable operation conditions (e.g., for very lean or low-temperature combustion). Chemiluminescence in the UV-Vis region fulfills all of these requirements, and it has been shown that it can be used to determine flame parameters such as stoichiometry and heat release quantitatively.

However, even though flame emissions have been studied for more than one century, important facts still remain unknown. Especially, reaction pathways leading to chemiluminescent species such as $\mathrm{OH}^{*}, \mathrm{CH}^{*}, \mathrm{C}_{2}^{*}$ and $\mathrm{CO}_{2}^{*}$ are still under debate and cannot be modeled with standard codes for flame simulation. In several cases, even the source species of spectral features observed in flames are unknown.

To investigate these processes in more detail, the cooperative research project "Chemiluminescence and Heat Release" has been started in 2006 with support of the Deutsche Forschungsgemeinschaft (DFG) under contract PAK 116. Seven individual projects, contributions from nine groups and institutions including the IWR and PCI at Heidelberg University, DLR Stuttgart, IVG of University of DuisburgEssen, PC1 at Bielefeld University, EKT at TU Darmstadt, PTB Braunschweig, TCP at Karlsruhe Institute of Technology and LTD at TU München. The project has been very

\footnotetext{
A. Brockhinke ( $\triangle)$

Physical Chemistry I, Bielefeld University, Universitätsstraße 25, 33615 Bielefeld, Germany

e-mail: andreas.brockhinke@uni-bielefeld.de

C. Schulz

IVG, Institute for Combustion and Gasdynamics, University of Duisburg-Essen, 47048 Duisburg, Germany

e-mail: christof.schulz@uni-due.de
}

successful and served as a platform for the exchange of ideas, technical and modeling resources on the subject under investigation. Several international meetings have been held during the course of the project, and guests from abroad shared their expertise on "chemiluminescence in flames". This special issue of Applied Physics B features selected papers from this research initiative.

This feature section of "Applied Physics B" is dedicated to show progress made in this field in the last years and highlight facets of the cooperative research project. Work on chemiluminescence is truly interdisciplinary, as it transcends the usual boundaries between applied and fundamental research fields. It involves the study of elementary formation reactions, detection of precursor species, analysis of the spectral properties of the chemiluminescence signal, tomographic methods for signal reconstruction, correlation of measured signals with results obtained by heat-release imaging, modeling of the reaction kinetics and measurements in practical combustors. In-situ measurements based on advanced laser-based diagnostics are of crucial importance for the investigation of the chemiluminescence phenomenon. Required trace species concentrations as well as highly spatially and temporally resolved information about species concentrations in turbulent flames, stationary flames and shock tubes are used throughout many of the papers presented here.

Kinetics data of the elementary reactions leading to chemiluminescent species are a prerequisite for any attempt to model and to really understand the origin of these emissions. Metehan Bozkurt et al. investigated the kinetics of $\mathrm{OH}^{*}$ and $\mathrm{CH}^{*}$ chemiluminescence in hydrocarbon oxidation behind reflected shock waves and determined new rate coefficients for several pertinent reactions. Shock-tube experiments have also been used in the work of Madeleine Kopp et al., who developed a first-generation kinetics model for 
the formation of $\mathrm{CO}_{2}^{*}$ and $\mathrm{CH}_{2} \mathrm{O}^{*}$, two chemiluminescent species which are hard to quantify due to their broad-band emissions.

A closer look at the spectroscopy has been taken in the work of Andreas Brockhinke et al. who report measured and modeled rotationally resolved spectra of all major chemiluminescent species and showed that re-absorption can dramatically change the spectral shape and the intensity of the measured signals. Patrick Nau et al. present measurements of the absolute number density of electronically excited species in flames. This allows quantitative comparison of the concentration of chemiluminescent species with model calculations, which has only rarely been presented before.

The quality of flame kinetics modeling can be assessed by detailed comparisons between experimental and modeled chemiluminescence species profiles. Binta Prabasena et al. present such measurements in counterflow nonpremixed flames with varying strain rate and fuel composition. Trupti Kathrotia et al. report similar measurements in one-dimensional low-pressure flames. Both investigations show that current flame models still need to be refined until they can accurately predict chemiluminescent species.

Acetylene is a molecule involved in important reaction chains leading to chemiluminescent species. Steven Wagner et al. developed a high-resolution TDLAS spectrometer that allows qualitative, calibration-free and spatially resolved measurements of this important species in flames.

Chemiluminescence is generated close to the flame front, which might stretch for considerable distances. This poses a problem, since standard optics provide only line-of-sightintegrated information, whereas spatially resolved information is required. Nikolay Anikin et al. developed a system of multiple telescopes which allows tomographic reconstruc- tion of the $2 \mathrm{D}-\mathrm{OH}^{*}$ chemiluminescence distribution in turbulent non-premixed flames. A different problem is optics is addressed by Cem Ertem et al. In many cases, beam steering will affect chemiluminescence signals. They used ray tracing in an unconfined non-premixed turbulent jet flame using large eddy simulation to assess these effects.

One of the intriguing properties of chemiluminescence is that its signals can be correlated with important flame parameters. Markus Röder et al. present localized heat release measurements by evaluating simultaneous $\mathrm{OH}$ and $\mathrm{CH}_{2} \mathrm{O}$ laser-induced fluorescence imaging in a swirl-stabilized $30 \mathrm{~kW}$ flame and show that correlations of heat release and spatially integrated $\mathrm{OH}^{*}$ chemiluminescence do exist.

Felix Güthe et al. present chemiluminescence investigations in even larger devices. Flame spectra and photomultiplier signals obtained in gas turbine power plants have been used for flame sensing and monitoring purposes as well as flame transfer function measurements. This allowed, e.g., to identify acoustic mode shapes which are required for the development of stable combustors.

The aforementioned contributions give a good cross section of research done in the field of "Chemiluminescence of Flames". The ongoing research will culminate in a detailed model of electronically excited species in flames and will allow a correlation of chemiluminescence signals with important flame parameters such as stoichiometry and heat release. Doubtless, these correlations will prove to be valuable for model-based prediction (MPR) models for the control of instationary combustion.

Acknowledgements We want to express our gratitude to the Editorin-Chief Frank Träger and Jutta Kaisig of the University of Kassel for their advice and support in preparing this special issue. Deutsche Forschungsgemeinschaft (DFG) has funded this work under contract PAK 116. 